\title{
Application of IEC61850 Protocol in Smart Substation
}

\author{
Huiqing Zhu \\ Jiangsu Huayuan Instrument and Meter Co., Ltd, 21110, Nanjing, China \\ ds16090311@126.com
}

Keywords: IEC61850; smart substation; smart device; SCD document

\begin{abstract}
This paper introduces the content and characteristics of IEC61850 standard, and the basic knowledge about the model of layered structure and the configuration files. In addition, the application skills of IEC61850 standard in a project are presented via the example of the SCD documenting and configuration. The contribution of this paper is to help the artisans and managers on the production line to understand the standard soon and improve their professional skills, so as to guarantee the security and stability of power system operation.
\end{abstract}

\section{Introduction}

Since the middle of 1990s, the layered distributed substation integrated automation system has been gradually promoted and applied in power system in our country. Unattended substations have been realized. The safety and the economy of substation operation and management have got great improvement. However, with the progress of technology and the improvement of operations, the conventional substation integrated automation system gradually exposed some disadvantages, such as poor interoperability between the secondary equipments and the hardness of sharing information, poor system scalability, safe hidden trouble in secondary loops, etc. From 2004 to 2008, our country committee of electric power standards tracked and translated IEC61850 series synchronously, and 14 standard booklets were converted to DL/T 860 series standard of electric power industry [1]. Based on the trend and technology as an opportunity to IEC 61850 standard in traditional substation automation communication protocol and the communication technology, based on the object-oriented modeling technology and future-oriented abstract communication service interface, quickly became the relay protection and substation automation, power quality, the important technology in the field of high voltage equipment condition monitoring and other support.

\section{Contents of IEC 61850 standard}

14 booklets of IEC 61850-1 to IEC 61850-10 formed IEC 61850 series standard, which are logically divided into five sections:

(1) System section: This category includes 5 blankets from IEC 61850-1 to IEC 61850-5. This section mainly introduces the starting point, the general requirements and standards of the standards set, as well as the system engineering management, system communication requirements and equipment model, etc.

(2) Configuration section: IEC 61850-6 rules description languages for substation intelligent electronic device configuration. The language called Substation Configuration description Language (SCL) to describe the relationship between electric power automation system and process regularly.

(3) Data model: This section mainly includes the IEC61850-7-3/4, which introduces the public data, compatible logical node and data classes defined by IEC 61850, from the angle of technical implementation, realizes the unity of the model for system communication information.

(4) Abstract communication service and mapping: This section mainly includes IEC 61850-7-1/2, IEC 61850-8-1 and IEC 61850-9-1/2. This section introduces the principle of the abstract communication service mapping to actual communication network, which realizes the unity of the communication services and the unity of the transmission process. 
(5) Test section: IEC 61850-10 stipulates the standard technology that implements conformance testing and specific measurement technology that is used when performance parameters are putted forward.

\section{The main characteristics of the IEC 61850 standard}

\subsection{Information classification}

IEC61850 defines the model of substation's three-tier communication interface, as shown in Fig.1. The automation system of substation is divided into three layers from the concept of physics and logic, namely the control layer, spacer layer and process layer.

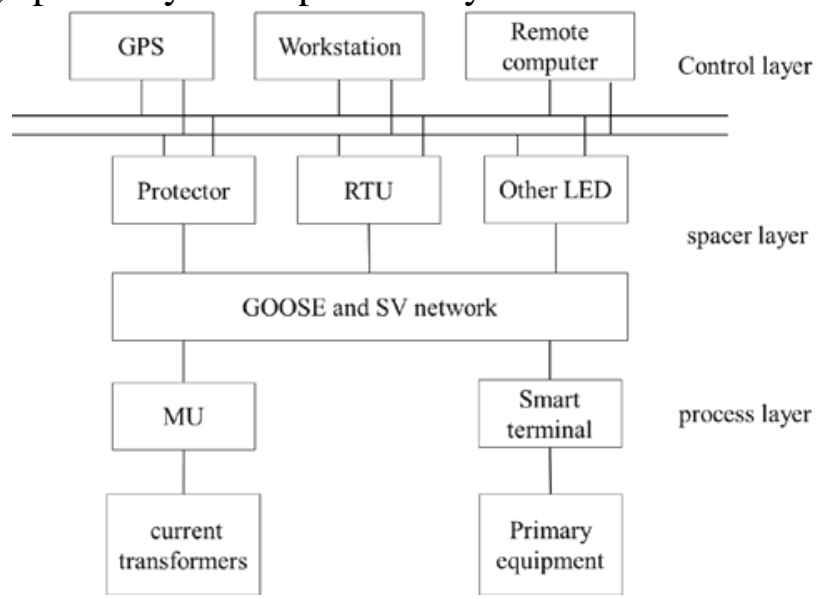

Fig.1 Three-tier configuration of substation in IEC61850

The devices of process layer comprise circuit breakers, disconnectors, current transformers, power transformer and other primary devices including their subordinate intelligent components (intelligent terminal of circuit breaker, current transformer merging unit etc.). The process layer is mainly responsible for the analog acquisition, input / output of state and the execution of operation control command and other functions related to devices.

The devices of spacer layer comprise protection device, control devices and the secondary auxiliary devices (such as digital fault recorders, network packet analyzer). The devices of spacer layer receive the process and the sampling information of the devices of process layer to realize the protection and control functions, and exchange information with the devices of station control layer through the network (such as sending up protection action signal and receiving switching on-off commands). The spacer layer network should also be able to transfer protection action coordination between the devices of spacer layer and joint locking information between the control devices (such as differential bus protection action taking far jump through the line protection, the closure to the remote control function of bus bar knife on line switch, etc.).

The devices of station control layer comprise monitoring hosts, operator stations, engineering stations, remote hosts, GPS timing devices, forming the monitoring and management center of the whole station. The station control layer provides human-computer interaction interface for managing operations of the primary devices and monitoring managing the secondary devices. At the same time, the devices of station control layer also communicate with the control center and the monitoring center through the power data network.

\subsection{The model of information is independent of communication protocol}

In order to adapt to the rapid development of communication technology, a comprehensive revision of the upper layer definition resulting from the progress of the bottom layer communication technology should be avoided. For communication service, IEC 61850 adopts the method of hierarchical definition. The service needed in the inner network communication of the substation's automation system is abstractly defined as Abstract Communication Service Interface (ACSI). Abstract Communication Service Interface is independent of specific the bottom layer network 
communication protocol and concrete network types. So it is universal and stable, and it meets the demand of the development of future communication technology well. In applications, ACSI needs to be mapped to concrete bottom layer network and protocol. This kind of mapping is called Specific Communication Service Mapping (SCSM). At present, IEC 61850-8-1 defines the mapping relationship between ACSI and MMS, and IEC 61850-9-2 defines the mapping between the process layer sampling service and Ethernet. The relationship between ACSI and SCSM is as shown in Fig. 2.

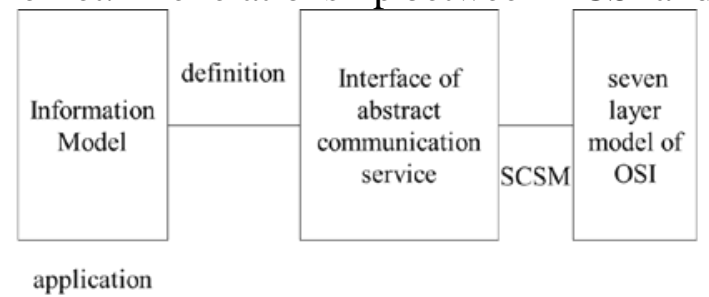

Fig.2 Relation between ACSI and SCSM

Obviously, with the progress of technology, there may be technologies and protocols which is more in line with the production characteristics of power network in the future. If the current MMS is replaced by another protocol according to the need in the future, we simply need to define a new SCSM and do not need to change various information model which have been defined in ACSI and IEC 61850. Similarly, if IEC 61850 needs expand and upgrade the information model, the communication protocols which have been adopted don't need to be changed. The communication style which ACSI is combined with SCSM brings enough expansibility for Smart Substation [2-3].

\subsection{Self description of data model}

Existing protocol standards use oriented signal, and identify with a dot. Its self descriptive is poor. The two sides of communication need to achieve agree and one-to-one correspond so that the equipment state can be correctly reflected. Once there is any change or adjustment, such as interval reconstruction or new interval, the data points in the database table from the monitor system to the dispatching end point must be made corresponding modification and each information point should be operated to verify its correctness, which is the work consuming a large quantity of manpower and material resources. While the IEC 61850 standard uses object-oriented self description method for information. In this kind of description, IEC 61850 establishes models for substation's automatic communication system and corresponding devices, functions and data. In the data source, it makes self description of data without prior agreement. The data is transferred with the description and the receiver can immediately build a database, so data engineering physical volumes don't need to be conversed for points and scales. The verification and database management and maintenance work are simplified.

\subsection{Object-oriented data unified modeling}

The IEC 61850 standard makes the information model have inheritance, reusability and other characteristics by using object-oriented technology. In simple terms, IEC 61850 standard states all the classes required in substation functions, and then defines all objects in the substation according to the actual situation when modeling. These objects connect and interact with each other to realize protection, monitoring, operation and other functions.

Object is a set which contains a number of attributes and their related services. Attribute and service are the two major elements of the object. Attribute is used to describe some features of it, while service is a function or behavior of object. An object can be very simple, and also can be very complex. That can be a concrete existence (such as a circuit breaker), and also can be abstract events which cannot be reached (such as distance protection logic). Taking a circuit breaker as a example, the circuit breaker is the object. The attribute of the circuit breaker is its position and the service of the circuit breaker is its switching operations.

Class is an abstract data type. Simple to say, class is a set and abstraction of similar objects. For example,\#1 \#2 and \#3 main transformer intelligent terminals are three different objects . They belong 
to the same type and their structure and characteristics are exactly the same so that they can be abstracted as the class of the main transformer intelligent terminal [4-6].

Through the introduction we can know that the relationship between class and object is the relationship between abstract concept and concrete examples. Class can be regarded as the templates or designs which generate object. Attributes and behaviors of the object are determined according to the class. In object-oriented design, a "class" is builded firstly and then it will be used to define some of the same type of objects. For example, intelligent terminal ICD files which belong to the same type can be defined for the use of multiple intervals to generate a plurality of different objects.

\section{Intelligent station system configurations}

\subsection{SCL documents intelligent station}

In order to provide a description of the unified, standard format for engineering tools of different vendors in the engineering practice of, and to make configuration description and engineering tools be interoperable between different vendors, IEC 61850-6 defines the substation configuration language SCL based on XML technology, which can describe substations first and secondary system by the way of independent standards, and can describe substation and intelligent electronic devices in the form of unified specification. IEC 61850-6 is an important part of IEC 61850 technology system and the important guarantee of IEC 61850 engineering implementation.

IEC 61850 regulates that the configuration files all adopt the SCL language which is object-oriented, and so all the configuration files have the same document structures. Documents should include five basic parts which are the header, substation, communication, intelligent electronic devices and data template.

\subsection{The classification configuration files}

When configurating files SCL language is used to describe generated files of substation equipment object model, to exchange configuration information of configuration tool between different manufacturers. Intelligent electronic devices from different vendors can communicate with each other to obtain needed information through the transmission of a series of configuration files. So the configuration files are the foundation of function realization of the digital substation system. IEC 61850 defines four types of configuration files:

ICD documents, namely IED Capability Description: Provided by device manufacturers for system integrators. This file describes the basic data model and service provided by IED. But the IED instance name and communication parameters are not included.

System Specification Description (SSD): This file describes first system structure of substation switching field and the associated logical nodes, and is included in the SCD file.

Substation Configuration Description (SCD) is only file in substation. This document describes the information of the instance configuration and communication parameters of all IED in total station, the connection between the IED and first system structure of substation. It is generated by system integrator for the time being. SCD file should contain version modification information, which clears modification time and modification version number, etc.

Configuration IED Description (CID): Each device has only one which is generated according to the IED information in SCD file by the device manufacturer.

\subsection{Configuration process of IEC 61850 project}

In the process of project implementation, system integrators provide system configuration tools. Tools each manufacturers use are different, which is no longer introduced in this paper. But configuration tools should be able to check the validity of the imported and exported configuration files. Generated configuration files should be able to pass Schema validation by SCL model. And can generate and maintain version number and revision number of configuration files. 
In principle, the configuration process import SSD and ICD files with the configuration tools for the system. According to the actual engineering situation, configuration person take use of the configuration tool for virtual connection, and make a correlation between first system and IED, implement total IED instantiation, and configurate communication parameters between IED. After the instantiation configuration in the system, total SCD configuration files should be exported, which are provided to clients, like the background monitor and remote host. CID files are extracted from the intelligent device, and bottomed in the IED. Finally all the devices are debugged, to verify the correctness of all the virtual terminal connections. But at the moment, due to lack of SSD files support, we adopt contact sheet of virtual the terminal contact form provided by design institute. This table shows all the secondary loop connection between the IED.

\section{Conclusions}

For the substation established with IEC 61850 standard, a large number of applications and the way of information transmission of intelligent electronic equipments are in fundamental change. These intelligent changes put forward the new requirements to knowledge and technology quality of relevant professionals. This paper systemic introduces theoretical knowledge, such as hierarchy model and configuration files of IEC 61850, and practice skills of making SCD files. The paper can help technical staffs in production understand and master the new standard as soon as possible, and improve their professional skills.

\section{References}

[1] CHEN Shu-yong, SONG Shu-fang. Smart Grid Technology. Power System Technology (2009), Vol.33, p. 1-7.

[2] LIU Xi-feng, HAN Zhen-feng, LI Ying. Application research of relationship between GOOSE message and SCD files. Power System Protection and Control (2013), Vol. 41, p. 95-100.

[3] HAO Xiao-guang. Function and Construction Policy Analysis on Intelligent Substation. HEBEI ELECTRIC POWER (2009), Vol. 11, p. 27-29.

[4] SONG Li-jun, WANG Ruo-xing, DI Jun-feng, etc. Analysis and implementation of GOOSE mechanism and its application in digital substation. Power System Protection and Control (2009), Vol. 37, p. 31-35.

[5] GAO Ya-dong, ZHU Bing-quan, LI Hui, etc. Application research on design method for "virtual terminal" of digital substation. Power System Protection and Control (2011), Vol. 39, p. 124-127.

[6] HE Lei, TIAN Xia, HAN Yong-jin, etc. Research on the testing of IEC 61850 configuration file in engineering. Power System Protection and Control (2011), Vol. 39, p. 147-150.

[7] ZHANG Pei-chao, GAO Xiang. System Architecture of Digitized Substation. Power System Technology (2006), Vol. 30, p. 73-77.

[8] CAO Nan, LI Gang, WANG Dong-qing. Key technology and construction methods of smart substation. Power System Protection and Control (2011), Vol. 39, p. 63-68. 